\title{
Phosphate and FGF23 in the renoprotective benefit of RAAS inhibition
}

\author{
Sophie de Seigneux ${ }^{1,2}$ and Pierre-Yves Martin ${ }^{1,2}$
}

1: Service of Nephrology, Department of Medical Specialties, University Hospital of Geneva and University of Geneva,

2: Laboratory of Nephrology, Department of Medicine Specialties and PHYME department, University of Geneva,

\section{Correspondance:}

Sophie de Seigneux

Service of Nephrology

University Hospital of Geneva

4 Rue Gabrielle Perret Gentil

1211 Genève 4

Sophie.deseigneux@hcuge.ch 


\section{Abstract}

Renin angiotensin-aldosterone system (RAAS) blockade is a mainstay of chronic kidney disease (CKD) treatment given its beneficial effects on proteinuria, nephroprotection, heart disease and global mortality.

The FGF23/Klotho/phosphate axis is crucial for phosphate excretion. During CKD, loss of Klotho, decreased phosphate excretion and FGF23 elevation are early events contributing both to renal disease progression and to cardiovascular complications. Experimental evidence suggests that Klotho replacement may improve renal and cardiovascular disease during CKD.

Recent evidence suggests that both RAAS activation and proteinuria decrease Klotho expression and lead to phosphate retention and FGF23 elevation. In opposition RAAS blockade may reverse Klotho loss during CKD in both experimental and human studies, with direct and indirect expected beneficial effects on the kidney and cardiovascular system. This effect of RAAS blockade on the FGF23/Klotho/phosphate axis may participate in explaining some of the beneficial effects of these drugs during CKD.

In this article we review the evidence linking RAAS blockade to modulation of the FGF23/Klotho/phosphate axis and the beneficial effects of these regulations. 


\section{Introduction}

ACE inhibitors and AT1 receptor Blockers (ARBs) display renoprotective effects in proteinuric diabetic and nondiabetic kidney diseases. Renin-angiotensin-aldosterone system (RAAS) blockade reduces proteinuria by decreasing intraglomerular pressure via a vasodilatory effect on the afferent and efferent glomerular arterioles ${ }^{1}$. This effect is significant (average $30 \%)$ and independent of the cause of proteinuria. ACE inhibitors' and ARB's nephroprotective properties on long term renal function have been demonstrated in several populations of proteinuric chronic kidney disease $(C K D)^{2-6}$ patients. In addition these drugs increase survival and improve heart function during left ventricular dysfunction ${ }^{7-10}$. Finally, RAAS blockade has been recently associated to decreased mortality in patients with CKD ${ }^{11}$. The FGF23/Klotho system regulates phosphate excretion and plays a major role in mineral metabolism ${ }^{12}$. Alterations of mineral metabolism are early events in CKD and contribute both to renal disease progression and to CKD extrarenal complications (mostly cardiovascular). Both proteinuria and RAAS have been described to interact with the FGF23/Klotho/phosphate system. In this article, we first introduce the FGF23/Klotho/phosphate system in CKD, then review the evidence linking proteinuria and RAAS to the FGF23/Klotho system and finally discuss the potential beneficial role of RAAS inhibition via FGF23/Klotho and phosphate regulation. 


\section{The FGF23/Klotho/Phosphate axis during chronic kidney disease (CKD)}

Major modifications of the FGF23/Klotho/phosphate axis occur already early in the course of CKD and influence both renal and extrarenal evolutions of the disease ${ }^{13,14}$. The anomalies of phosphate excretion are usually defined as CKD -mineral and bone disease (CKD-MBD). In general, the more advanced the CKD, the more severe the CKD-MBD. Epidemiologically, there are clear evidence linking perturbations of mineral metabolism to cardiovascular mortality and renal disease progression. Phosphatemia is indeed predictive of cardiovascular mortality both in patients with altered or preserved renal function, even when phosphate levels are still within the normal range ${ }^{15-20}$. Phosphate retention may also directly enhance CKD progression ${ }^{21,22}$. FGF23 elevation is a marker of increased mortality in several population including CKD patients, kidney allograft patients, dialysis patients and the general population ${ }^{23-28}$. FGF23 levels are predictive of renal function decline in CKD patients ${ }^{26}$. Klotho measurements are not highly reproducible in human currently and large epidemiological studies linking its expression to cardiovascular or renal prognosis are lacking, but experiments clearly point to a key role of this protein in mediating cardiovascular and renal protection $^{14,29-35}$.

The pathophysiology of CKD-MBD is still a matter of debate. The current understanding of CKD-MDB is that Klotho loss is a very early event in different types of CKD ${ }^{13,14,36-38}$. Klotho is a transmembranous protein expressed mainly in the distal nephron, and at lower levels in other segments of the nephron and the parathyroid gland ${ }^{13,14}$. Klotho was first identified as an anti-aging protein and its complete deletion in mice induces early mortality with a multisystemic disease including massive cardiovascular calcifications, bone disease, skin atrophy and lung emphysema among others ${ }^{39}$. Targeted renal Klotho deletion induces an 
extrarenal phenotype similar to that observed in the Klotho null mouse, indicating the major importance of renal Klotho in the cardiovascular calcifications and phosphate retention of Klotho deficient animals ${ }^{40,41}$. Vascular calcifications of Klotho null mice can in part be rescued by lowering phosphate retention through different interventions, which also improve lifespan, demonstrating that phosphate accumulation is as an important factor in this cardiovascular phenotype ${ }^{42}$. Indeed Klotho is a co-factor for FGF23, a major phosphaturic hormone produced in the bone ${ }^{12}$, and its presence is mandatory for FGF23 binding on its receptor and being biologically active. In addition, soluble Klotho directly acts on some phosphate and calcium transporters and induces phosphaturia ${ }^{43}$. Klotho loss therefore impairs phosphate excretion and has major detrimental effects through this pathway given the vascular, an potential renal, toxicity of phosphate ${ }^{44,45}$. In addition, Klotho loss may have phosphate independent effects ${ }^{14,29}$. Experimentally Klotho replacement rescues the progression of nephropathies ${ }^{33,34,46}$. This effect may be mediated by several mechanisms among which the repression of the Wnt/ $\beta$-Catenin profibrotic signaling pathway by Klotho may play an important role ${ }^{35}$. Finally, during experimental CKD, soluble Klotho administration protects against CKD associated cardiovascular complications ${ }^{31,32}$. This likely relies on the phosphaturic properties of Klotho, but also on direct antifibrotic properties of the protein, via regulation of the cardiac Smad pathway for example ${ }^{29}$.

During CKD, circulating and renal Klotho decrements precedes FGF23 elevation ${ }^{47} 37$. The mechanisms underlying exaggerated FGF23 secretion in CKD are not known. Increased FGF23 secretion may be consecutive to Klotho loss or to a transient positive phosphate balance due to GFR loss ${ }^{12,48}$. Alternative explanation for the high FGF23 levels of CKD may be an exaggerated secretion by bone cells via unknown factors, or FGF23 secretion by diseased kidneys ${ }^{49,50}$. FGF23 then stimulates phosphate excretion and decreases 1.25 
vitamin $D$ hydroxylation, maintaining phosphatemia until late stages of $C K D$, and potentially even decreasing it at initial stages ${ }^{51}$. PTH secretion is then stimulated by the decreased levels of 1.25 vitamin $D^{52}$. Maintenance of a near-normal phosphatemia in advancing CKD therefore results from the elevation of phosphaturic hormones. It is however likely that phosphate body stores increase in a much faster way than phosphatemia during progressive $\mathrm{CKD}^{48}$, although no clear demonstration of this exists since no reliable measurements of phosphate stores are available. One of the explanations for the excellent predictive value of FGF23 levels for vascular complications in CKD may therefore be its role as a marker of Klotho loss, early renal lesions and/or elevated total phosphate body stores.

Recent evidence also suggest that FGF23 may possess direct cardiotoxic properties since this hormone induces cardiac hypertrophy when injected to mice ${ }^{53}$, and its level correlates to left ventricular hypertrophy in human subjects, mostly in the context of CKD ${ }^{53,54}$. FGF23 cardiotoxicity appears to be mediated by the stimulation of the cardiac receptor FGFR4 through high level of FGF23 ${ }^{55}$. Further recent experimental evidences also show that FGF23 stimulates sodium reabsorption and volume expansion through NCC transporters phosphorylation in the distal nephron ${ }^{56}$, further possibly linking FGF23 to cardiac hypertrophy. One study suggested that FGF23 blockade may decreases heart hypertrophy in an animal model of $\mathrm{CKD}^{57}$. Similarly FGFR4 blockade attenuates cardiac hypertrophy ${ }^{55}$. However, the human impact of these observations is still to be demonstrated and the relative roles of FGF23, phosphate elevation versus Klotho deficiency in uremic cardiopathy are still debated ${ }^{29}$. Indeed, in a recent experiment, FGF23 was associated to cardiac remodeling only in the presence of Klotho deficiency ${ }^{29}$. Finally, despite the potential deleterious cardiac effects of FGF23, its inhibition leads to rapid calcifications progression and enhanced mortality during experimental CKD, demonstrating that the phosphaturic 
properties of this factor are clearly protective and needed for survival during $C K D^{58}$, despite its potential role on cardiac hypertrophy.

Altogether, current evidence suggests that phosphate accumulation secondary to Klotho and GFR loss, is pivotal in the pathophysiology of cardiovascular disease in CKD. Klotho loss appears to be an early and important event directly linking CKD to vascular calcifications and cardiac hypertrophy in a phosphate dependent and probably also independent manner. Finally, FGF23, although critically needed to maintain phosphate excretion, may also display cardiotoxic properties.

\section{RAAS inhibition, proteinuria and FGF23/Klotho/phosphate axis}

ACE inhibitors and ARBs alleviate the deleterious effects of RAAS activation. Their renoprotective effect is demonstrated in diabetic and non diabetic proteinuric nephropathies and appears to result mainly from proteinuria reduction but also from inhibition of Angiotensin II production or activity ${ }^{59-62}$. In addition, RAAS blockade is beneficial in heart failure through several effects ${ }^{63}$. Finally, recent evidence demonstrates that ACE inhibition decreases mortality in CKD patients ${ }^{11}$.

There are several potential axes of interaction between RAAS and FGF23/Klotho/phosphate axis in CKD. Some of the beneficial effects of ACE inhibition and ARBs may therefore rely on the regulation of this axis.

Angiotensin II has been demonstrated to downregulate kidney Klotho expression in animal models, whereas Klotho substitution ameliorates angiotensin II-induced renal damage ${ }^{33}$. Klotho downregulation by angiotensin II appears to be independent of blood pressure but dependent on AT1 receptor activation ${ }^{64}$. Furthermore, Klotho expression decreases during 
dehydration with a potential direct effect of $A D H$ and aldosterone on its expression ${ }^{65}$. Thus activation of RAAS appears to reduce kidney Klotho expression. In reverse, ACE inhibition or AT1R blockade in experimental CKD increases Klotho expression in cyclosporin toxicity and in spontaneously hypertensive rats ${ }^{64,66}$. This effect appears to be independent of proteinuria reduction, since these experimental models do not harbor important glomerular proteinuria. Similar observations are made using a direct renin inhibitor in ischemic nephropathy ${ }^{67}$. Conversely, recent evidence demonstrates that Klotho also regulates RAAS activation ${ }^{68}$. Indeed, Klotho represses Wnt/ $\beta$ Catenin pathway, which in turns targets RAAS genes ${ }^{35,69}$. The crosstalk between the two systems appears therefore to be very active and complex.

Recent evidence also link proteinuria to altered Klotho expression and tubular handing of phosphate. Indeed, in a large cohort of CKD patients, we have demonstrated that proteinuria was associated to higher phosphate levels despite higher FGF23 and that this effect was independent of $\mathrm{GFR}^{70}$. This suggests that proteinuria may induce a resistance to FGF23 biological activity. In nephrotic children, we have observed that transient nephrotic syndrome was related to impaired tubular phosphate excretion despite high FGF23 levels. We did also observe that proteinuria regulates Klotho expression in animal models of proteinuria, leading to FGF23 resistance and $\mathrm{NaPi2a}$ relative overexpression. This was in line with previous observation including ours that albumin is uptaken in the proximal and distal nephron $^{71}$. In a cohort of CKD children, those with glomerular disease, and therefore higher albuminuria, had higher phosphate and FGF23 levels than children with tubulo-interstitial disease $^{72}$. Two other reports in children confirm that nephrotic syndrome is associated to increased serum phosphate levels, and phosphate levels correlated to elevated pulse wave pressure in a serie of 42 nephrotic children ${ }^{73,74}$. In IgA nephropathy, albuminuria was also associated to higher FGF23 levels independently of renal function ${ }^{75}$. Finally a recent study 
demonstrated that proteinuria was inversely correlated to circulating Klotho levels in CKD patients, more accurately than to glomerular filtration rate ${ }^{76}$. Altogether proteinuria appears to induce phosphate retention and FGF23 elevation independently of decreased glomerular filtration potentially in part via Klotho downregulation.

Oppositely, ACE inhibition and AT1 receptor blockade increase Klotho expression during proteinuric CKD. In a model of diabetic nephropathy, ACE inhibition was efficient to reduce both systemic and renal expressions of FGF23 together with increasing Klotho expression and normalizing phosphate level and excretion ${ }^{50}$. This effect might be mediated by a direct effect on angiotensin II and aldosterone, or by an indirect effect through reduction of proteinuria in CKD, since proteinuria was decreased by ACE inhibition in this model. In a post hoc analysis of 33 patients suffering from type 2 diabetes randomized in crossover to losartan and quinalapril, it was also demonstrated that losartan treatment increased circulating Klotho levels, whereas quinalapril did not. Interestingly in this study, losartan decreased proteinuria whereas quinalapril was inefficient. These discrepancies between the two treatments may very likely be related to the non maximal dose of quinalapril used, since no effect on blood pressure was also observed ${ }^{77}$. Another recent study on 76 patients with diabetic kidney disease showed that 24 months treatment with valsartan and hydrochlorothiazide increased circulating Klotho and decreased phosphate levels significantly compared to amlodipine treatment ${ }^{78}$. Very interestingly, albuminuria and aortic pulse wave velocity declined significantly in valsartan/hydrochlorothiazide group, despite similar effects on blood pressure. The effect of valsartan/hydrochlorothiazide on Klotho and phosphate might thus be related to either an effect on proteinuria or to a direct effect on RAAS inhibition. FGF23 levels were not significantly altered in this setting, although a decrease would have been expected. There is currently no good explanation for the absence 
of FGF23 decrease in this context, although eGFR was altered by the therapy. Similar observations were made in children: a post-hoc analysis of the ESCAPE trial randomizing 167 children with CKD showed that ACE inhibition was associated to increased serum Klotho and decreased proteinuria after 8 months of follow up ${ }^{79}$. ACE inhibition surprisingly increased FGF23 in this trial and did not modify phosphate level. Again there is no explanation for the increase in FGF23, but as eGFR declined during the 8 months follow-up, the net effects on phosphate and FGF23 may be complex to interpret. Of interest, the presence of a high phosphate in the REIN trial was associated independently of proteinuria to a worse renal outcome $^{80}$. Patients with high serum phosphate displayed less renoprotective effects of ACE inhibitors. A similar observation was made in another CKD cohort where high phosphate levels were also predictive of lower renoprotective effects of RAAS inhibition ${ }^{81}$. This indicates that an interaction between phosphate and the RAA system exists and that renoprotective effects may also be dependent on regulation of Klotho by ACE inhibitors.

Altogether, experimental and clinical studies clearly show that RAAS inhibition restores in part Klotho expression in proteinuric CKD. These observations are important since in animals models, increasing soluble Klotho decreases cardiovascular lesions associated to $C K D^{32}$. In addition, Klotho replacement improves renal lesions in experimental CKD ${ }^{46}$. These benefits are likely mediated through the potent phosphaturic effect of Klotho, directly and as FGF23 cofactor, but also via possible direct effects, such as WNT/ $\beta$ Cathenin or smad pathway regulation for kidney and heart fibrosis ${ }^{35}$. In addition, Klotho by itself may contribute to suppress the RAAS ${ }^{68}$. The beneficial effects of ACE inhibition on the kidney and the heart may therefore partly rely on proteinuria dependent and/or independent regulation of Klotho expression during CKD. ACE inhibitors are the first drugs used in CKD patients to be demonstrated to increase Klotho levels repeatedly, with benefic effects on FGF23 and 
phosphate levels in some studies, all factors associated to cardiovascular and renal prognosis

during CKD. Restoration of Klotho may participate to the well known beneficial effects of RAAS blockade on kidney disease progression, heart failure and mortality. 


\section{Conclusions and perspectives}

There are several points of potential interactions between RAA system and FGF23/Klotho axis. RAAS blockade appears to rescue Klotho downregulation observed in various types of CKD models and in humans. This effect may be linked to a direct consequence of RAAS inhibition and/or to reduction of proteinuria and its subsequent improvement in Klotho expression and tubular FGF23 resistance. These observations are important since FGF23/Klotho axis dysregulation appears to play important roles in CKD progression as well as CKD-related heart and vascular diseases. The beneficial effect of ACE inhibition on kidney, heart disease and mortality during CKD may therefore partly rely on these newly observed effects on Klotho expression. Finally, there is an ongoing quest on drugs that could regulate the FGF23/Klotho/phosphate system in CKD and may reverse renal disease progression and decrease extrarenal complications. The recent observations described in this review suggest that RAAS blockers are the only drugs available currently for human use to have a beneficial effect on this axis. Although further data is needed to ascertain the links we draw here, evidence is pointing to an interaction between RAAS blockade and the FGF23/Klotho/phosphate axis.

\section{Acknowledgments:}

SdS is funded by grants from the Swiss National Science foundation, the national center for competence in Research NCCR. Kidney.ch, the Von Meissner Foundation and Schmidheiny Foundation 


\section{References}

1. Anderson, S., Rennke, H.G., Garcia, D.L. \& Brenner, B.M. Short and long term effects of antihypertensive therapy in the diabetic rat. Kidney Int 36, 526-536 (1989).

2. Remuzzi, G. \& Benigni, A. Progression of proteinuric diabetic and nondiabetic renal diseases: a possible role for renal endothelin. Kidney Int Supp/ 58, S66-68 (1997).

3. Remuzzi, G. \& Ruggenenti, P. Overview of randomised trials of ACE inhibitors. Lancet 368, 555-556 (2006).

4. Ruggenenti, P., et al. Renoprotective properties of ACE-inhibition in non-diabetic nephropathies with non-nephrotic proteinuria. Lancet 354, 359-364 (1999).

5. Ruggenenti, P., et al. Blood-pressure control for renoprotection in patients with non-diabetic chronic renal disease (REIN-2): multicentre, randomised controlled trial. Lancet 365, 939-946 (2005).

6. Ruggenenti, P., et al. Renal function and requirement for dialysis in chronic nephropathy patients on long-term ramipril: REIN follow-up trial. Gruppo Italiano di Studi Epidemiologici in Nefrologia (GISEN). Ramipril Efficacy in Nephropathy. Lancet 352, 1252-1256 (1998).

7. Effect of enalapril on survival in patients with reduced left ventricular ejection fractions and congestive heart failure. The SOLVD Investigators. N Engl J Med 325, 293-302 (1991).

8. Effect of enalapril on mortality and the development of heart failure in asymptomatic patients with reduced left ventricular ejection fractions. The SOLVD Investigattors. $N$ Engl J Med 327, 685-691 (1992).

9. Swedberg, K., et al. Effects of the early administration of enalapril on mortality in patients with acute myocardial infarction. Results of the Cooperative New Scandinavian Enalapril Survival Study II (CONSENSUS II). N Engl J Med 327, 678-684 (1992).

10. von Lueder, T.G. \& Krum, H. RAAS inhibitors and cardiovascular protection in large scale trials. Cardiovascular drugs and therapy / sponsored by the International Society of Cardiovascular Pharmacotherapy 27, 171-179 (2013).

11. Molnar, M.Z., et al. Angiotensin-converting enzyme inhibitor, angiotensin receptor blocker use, and mortality in patients with chronic kidney disease. J Am Coll Cardiol 63, 650-658 (2014).

12. Hu, M.C., Shiizaki, K., Kuro, O.M. \& Moe, O.W. Fibroblast growth factor 23 and klotho: physiology and pathophysiology of an endocrine network of mineral metabolism. Annu Rev Physiol 75, 503-533 (2013).

13. Hu, M.C., Kuro-o, M. \& Moe, O.W. Klotho and chronic kidney disease. Contrib Nephrol 180, 47-63 (2013).

14. Hu, M.C., Kuro-o, M. \& Moe, O.W. Renal and extrarenal actions of Klotho. Semin Nephrol 33, 118-129 (2013).

15. Kestenbaum, B., et al. Serum phosphate levels and mortality risk among people with chronic kidney disease. J Am Soc Nephrol 16, 520-528 (2005).

16. Tonelli, M., Sacks, F., Pfeffer, M., Gao, Z. \& Curhan, G. Relation between serum phosphate level and cardiovascular event rate in people with coronary disease. Circulation 112, 26272633 (2005).

17. Dhingra, R., et al. Relations of serum phosphorus levels to echocardiographic left ventricular mass and incidence of heart failure in the community. European journal of heart failure 12, 812-818 (2010).

18. Dhingra, R., et al. Relations of serum phosphorus and calcium levels to the incidence of cardiovascular disease in the community. Arch Intern Med 167, 879-885 (2007).

19. Foley, R.N., Collins, A.J., Ishani, A. \& Kalra, P.A. Calcium-phosphate levels and cardiovascular disease in community-dwelling adults: the Atherosclerosis Risk in Communities (ARIC) Study. American heart journal 156, 556-563 (2008). 
20. Dominguez, J.R., et al. Relationships between serum and urine phosphorus with all-cause and cardiovascular mortality: the Osteoporotic Fractures in Men (MrOS) Study. Am J Kidney Dis 61, 555-563 (2013).

21. Kuro, O.M. A phosphate-centric paradigm for pathophysiology and therapy of chronic kidney disease. Kidney international supplements 3, 420-426 (2013).

22. Kuro-o, M. Klotho, phosphate and FGF-23 in ageing and disturbed mineral metabolism. Nat Rev Nephrol 9, 650-660 (2013).

23. Gutierrez, O.M., et al. Fibroblast growth factor 23 and mortality among patients undergoing hemodialysis. N Engl J Med 359, 584-592 (2008).

24. Wolf, M., et al. Elevated fibroblast growth factor 23 is a risk factor for kidney transplant loss and mortality. J Am Soc Nephrol 22, 956-966 (2011).

25. Arnlov, J., et al. Higher fibroblast growth factor-23 increases the risk of all-cause and cardiovascular mortality in the community. Kidney Int 83, 160-166 (2013).

26. Fliser, D., et al. Fibroblast growth factor 23 (FGF23) predicts progression of chronic kidney disease: the Mild to Moderate Kidney Disease (MMKD) Study. J Am Soc Nephrol 18, $2600-$ 2608 (2007).

27. Parker, B.D., et al. The associations of fibroblast growth factor 23 and uncarboxylated matrix Gla protein with mortality in coronary artery disease: the Heart and Soul Study. Ann Intern Med 152, 640-648 (2010).

28. Brandenburg, V.M., et al. Fibroblast growth factor 23 (FGF23) and mortality: the Ludwigshafen Risk and Cardiovascular Health Study. Atherosclerosis 237, 53-59 (2014).

29. Hu, M.C., et al. Klotho and phosphate are modulators of pathologic uremic cardiac remodeling. J Am Soc Nephrol 26, 1290-1302 (2015).

30. Hu, M.C., et al. Klotho deficiency causes vascular calcification in chronic kidney disease. J Am Soc Nephrol 22, 124-136 (2011).

31. Xie, J., et al. Cardioprotection by Klotho through downregulation of TRPC6 channels in the mouse heart. Nature communications 3, 1238 (2012).

32. Xie, J., Yoon, J., An, S.W., Kuro-o, M. \& Huang, C.L. Soluble Klotho Protects against Uremic Cardiomyopathy Independently of Fibroblast Growth Factor 23 and Phosphate. J Am Soc Nephrol 26, 1150-1160 (2015).

33. Mitani, $\mathrm{H}_{\text {., }}$ et al. In vivo klotho gene transfer ameliorates angiotensin II-induced renal damage. Hypertension 39, 838-843 (2002).

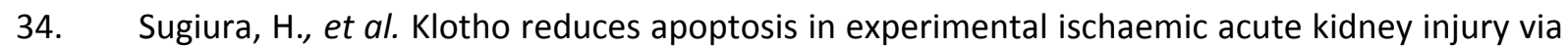
HSP-70. Nephrol Dial Transplant 25, 60-68 (2010).

35. Zhou, L., Li, Y., Zhou, D., Tan, R.J. \& Liu, Y. Loss of Klotho contributes to kidney injury by derepression of Wnt/beta-catenin signaling. J Am Soc Nephrol 24, 771-785 (2013).

36. Hu, M.C., et al. Klotho deficiency is an early biomarker of renal ischemia-reperfusion injury and its replacement is protective. Kidney Int (2010).

37. Pavik, I., et al. Secreted Klotho and FGF23 in chronic kidney disease Stage 1 to 5: a sequence suggested from a cross-sectional study. Nephrol Dial Transplant 28, 352-359 (2013).

38. Seiler, S., et al. Plasma Klotho is not related to kidney function and does not predict adverse outcome in patients with chronic kidney disease. Kidney Int 83, 121-128 (2013).

39. Kuro-o, M., et al. Mutation of the mouse klotho gene leads to a syndrome resembling ageing. Nature 390, 45-51 (1997).

40. Shimada, T., et al. Targeted ablation of Fgf23 demonstrates an essential physiological role of FGF23 in phosphate and vitamin D metabolism. J Clin Invest 113, 561-568 (2004).

41. Lindberg, K., et al. The kidney is the principal organ mediating klotho effects. J Am Soc Nephrol 25, 2169-2175 (2014).

42. Kuro, O.M. Phosphate and Klotho. Kidney Int Suppl, S20-23 (2011).

43. Hu, M.C., et al. Klotho: a novel phosphaturic substance acting as an autocrine enzyme in the renal proximal tubule. FASEB J 24, 3438-3450 (2010). 
44. Shanahan, C.M. Mechanisms of vascular calcification in CKD-evidence for premature ageing? Nat Rev Nephrol 9, 661-670 (2013).

45. Shroff, R., Long, D.A. \& Shanahan, C. Mechanistic insights into vascular calcification in CKD. J Am Soc Nephrol 24, 179-189 (2013).

46. Haruna, Y., et al. Amelioration of progressive renal injury by genetic manipulation of Klotho gene. Proc Natl Acad Sci U S A 104, 2331-2336 (2007).

47. Shimamura, Y., et al. Serum levels of soluble secreted alpha-Klotho are decreased in the early stages of chronic kidney disease, making it a probable novel biomarker for early diagnosis. Clin Exp Nephrol 16, 722-729 (2012).

48. Huang, C.L. \& Moe, O.W. Clinical assessment of phosphorus status, balance and renal handling in normal individuals and in patients with chronic kidney disease. Current opinion in nephrology and hypertension 22, 452-458 (2013).

49. Wolf, M. Update on fibroblast growth factor 23 in chronic kidney disease. Kidney Int 82, 737747 (2012).

50. Zanchi, C., et al. Renal expression of FGF23 in progressive renal disease of diabetes and the effect of ACE inhibitor. PLoS One 8, e70775 (2013).

51. Isakova, T., et al. Fibroblast growth factor 23 is elevated before parathyroid hormone and phosphate in chronic kidney disease. Kidney Int 79, 1370-1378 (2011).

52. Isakova, T. \& Wolf, M.S. FGF23 or PTH: which comes first in CKD ? Kidney Int 78, 947-949 (2010).

53. Faul, C., et al. FGF23 induces left ventricular hypertrophy. J Clin Invest 121, 4393-4408 (2011).

54. Jovanovich, A., et al. Fibroblast growth factor 23 , left ventricular mass, and left ventricular hypertrophy in community-dwelling older adults. Atherosclerosis 231, 114-119 (2013).

55. Grabner, A., et al. Activation of Cardiac Fibroblast Growth Factor Receptor 4 Causes Left Ventricular Hypertrophy. Cell metabolism 22, 1020-1032 (2015).

56. Andrukhova, O., et al. FGF23 regulates renal sodium handling and blood pressure. EMBO Mol Med 6, 744-759 (2014).

57. Di Marco, G.S., et al. Treatment of established left ventricular hypertrophy with fibroblast growth factor receptor blockade in an animal model of CKD. Nephrol Dial Transplant 29, 2028-2035 (2014).

58. Shalhoub, V., et al. FGF23 neutralization improves chronic kidney disease-associated hyperparathyroidism yet increases mortality. J Clin Invest 122, 2543-2553 (2012).

59. Ruster, C. \& Wolf, G. Renin-angiotensin-aldosterone system and progression of renal disease. J Am Soc Nephrol 17, 2985-2991 (2006).

60. Cravedi, P., Ruggenenti, P. \& Remuzzi, G. Which antihypertensive drugs are the most nephroprotective and why? Expert opinion on pharmacotherapy 11, 2651-2663 (2010).

61. Ruggenenti, P., Cravedi, P. \& Remuzzi, G. The RAAS in the pathogenesis and treatment of diabetic nephropathy. Nat Rev Nephrol 6, 319-330 (2010).

62. Ruggenenti, P., Cravedi, P. \& Remuzzi, G. Mechanisms and treatment of CKD. J Am Soc Nephrol 23, 1917-1928 (2012).

63. Shearer, F., Lang, C.C. \& Struthers, A.D. Renin-angiotensin-aldosterone system inhibitors in heart failure. Clinical pharmacology and therapeutics 94, 459-467 (2013).

64. Tang, R., et al. Fosinopril and losartan regulate klotho gene and nicotinamide adenine dinucleotide phosphate oxidase expression in kidneys of spontaneously hypertensive rats. Kidney \& blood pressure research 34, 350-357 (2011).

65. Tang, C., et al. Downregulation of Klotho expression by dehydration. Am J Physiol Renal Physiol 301, F745-750 (2011).

66. Yoon, H.E., et al. Angiotensin II blockade upregulates the expression of Klotho, the antiageing gene, in an experimental model of chronic cyclosporine nephropathy. Nephrol Dial Transplant 26, 800-813 (2011).

67. Sun, C.Y., et al. Aliskiren reduced renal fibrosis in mice with chronic ischemic kidney injury-beyond the direct renin inhibition. Hypertens Res 35, 304-311 (2012). 
68. Zhou, L., et al. Klotho Ameliorates Kidney Injury and Fibrosis and Normalizes Blood Pressure by Targeting the Renin-Angiotensin System. Am J Pathol 185, 3211-3223 (2015).

69. Zhou, L., et al. Multiple genes of the renin-angiotensin system are novel targets of Wnt/betacatenin signaling. J Am Soc Nephrol 26, 107-120 (2015).

70. de Seigneux, S., et al. Proteinuria Increases Plasma Phosphate by Altering Its Tubular Handling. J Am Soc Nephrol 26, 1608-1618 (2015).

71. Dizin, E., et al. Albuminuria induces a pro-inflammatory and pro-fibrotic response in cortical collecting duct cells via the 24p3 receptor. Am J Physiol Renal Physiol (2013).

72. Portale, A.A., et al. Disordered FGF23 and mineral metabolism in children with CKD. Clin J Am Soc Nephrol 9, 344-353 (2014).

73. Feinstein, S., Becker-Cohen, R., Rinat, C. \& Frishberg, Y. Hyperphosphatemia is prevalent among children with nephrotic syndrome and normal renal function. Pediatr Nephrol 21, 1406-1412 (2006).

74. Sexton, D.J., Kinsella, S.M. \& Eustace, J.A. Serum phosphate varies with degree of proteinuria in nephrotic syndrome and is associated with elevated pulse wave velocity. J Nephrol 26, 540-548 (2013).

75. Lundberg, S., et al. FGF23, albuminuria, and disease progression in patients with chronic IgA nephropathy. Clin J Am Soc Nephrol 7, 727-734 (2012).

76. Hage, V., et al. In chronic kidney disease, serum alpha-Klotho is related to serum bicarbonate and proteinuria. Journal of renal nutrition : the official journal of the Council on Renal Nutrition of the National Kidney Foundation 24, 390-394 (2014).

77. Lim, S.C., Liu, J.J., Subramaniam, T. \& Sum, C.F. Elevated circulating alpha-klotho by angiotensin II receptor blocker losartan is associated with reduction of albuminuria in type 2 diabetic patients. Journal of the renin-angiotensin-aldosterone system : JRAAS 15, 487-490 (2014).

78. Karalliedde, J., Maltese, G., Hill, B., Viberti, G. \& Gnudi, L. Effect of renin-angiotensin system blockade on soluble Klotho in patients with type 2 diabetes, systolic hypertension, and albuminuria. Clin J Am Soc Nephrol 8, 1899-1905 (2013).

79. Shroff, R., et al. Normal 25-Hydroxyvitamin D Levels Are Associated with Less Proteinuria and Attenuate Renal Failure Progression in Children with CKD. J Am Soc Nephrol (2015).

80. Zoccali, C., et al. Phosphate may promote CKD progression and attenuate renoprotective effect of ACE inhibition. J Am Soc Nephrol 22, 1923-1930 (2011).

81. Jovanovich, A.J., et al. Mineral Metabolites, Angiotensin II Inhibition and Outcomes in Advanced Chronic Kidney Disease. Am J Nephrol 42, 361-368 (2015).

\section{Figure 1:}

Schematic simplified overview of mineral metabolism adaptations during chronic kidney disease. 

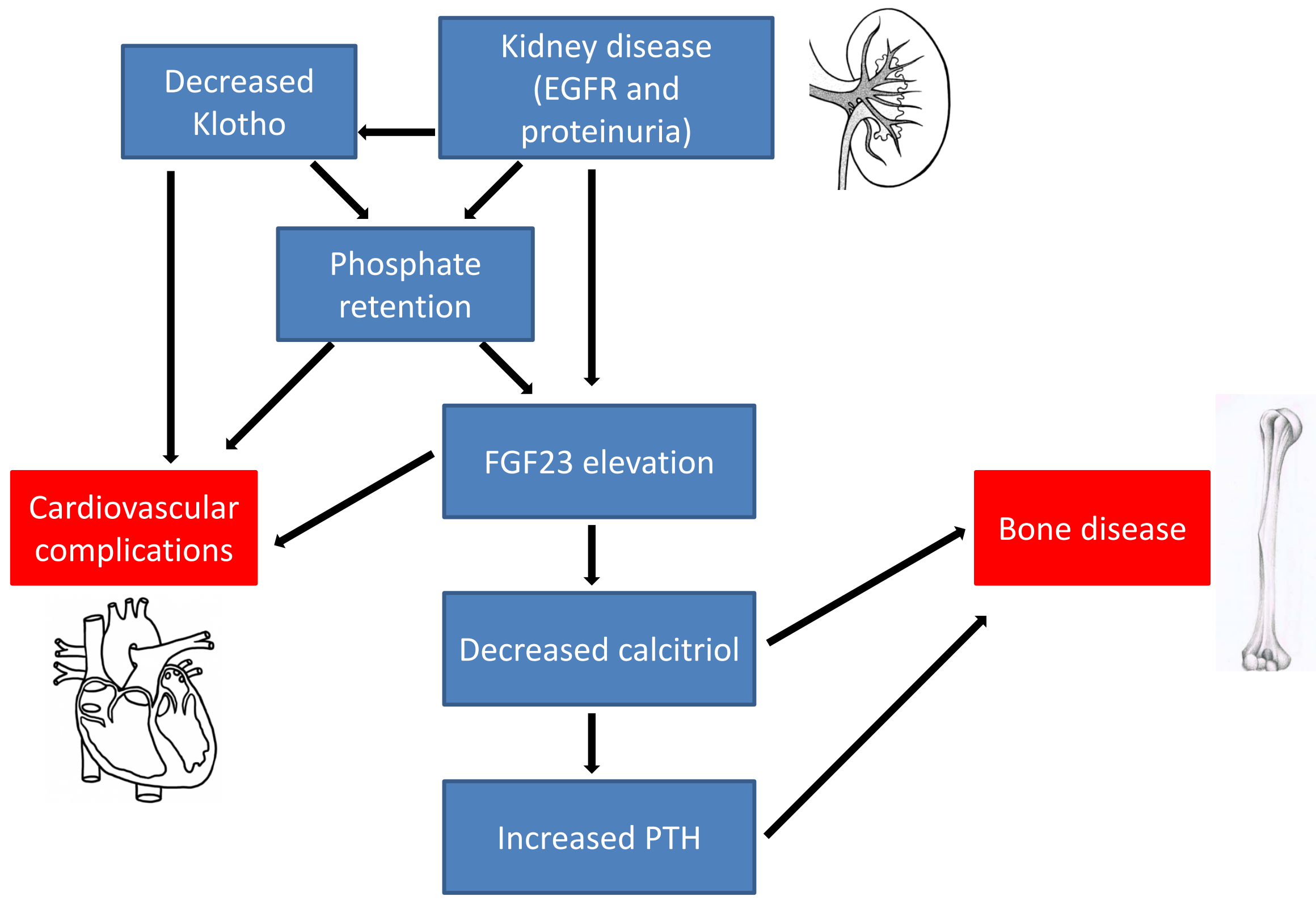


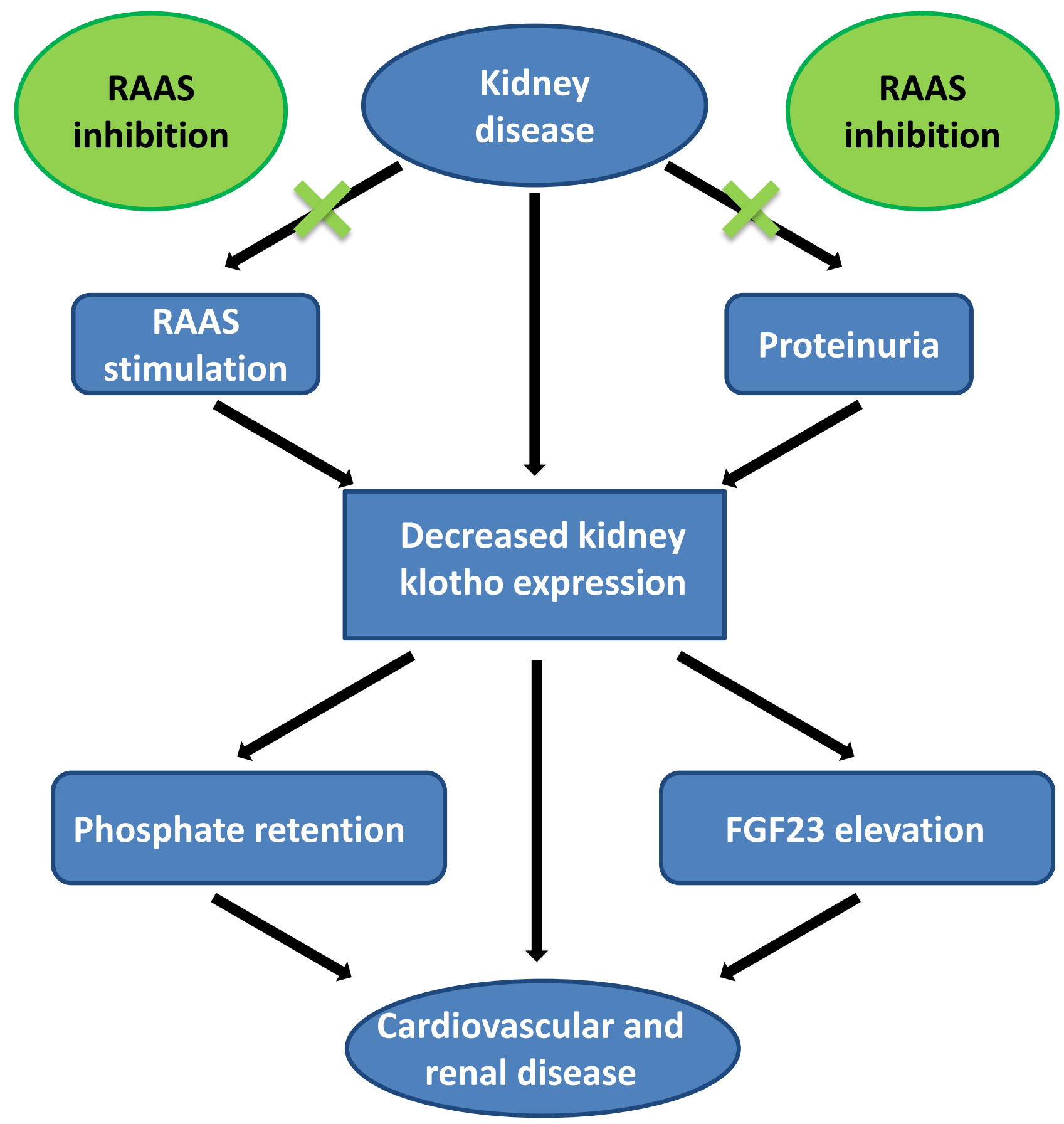

